\title{
REMARK ON DISCRETE SUBGROUPS ${ }^{1}$
}

\author{
JOSEPH A. WOLF
}

To A. A. Albert on his sixty-fifth birthday

\begin{abstract}
One wants to know the extent to which a locally compact group $G$ is determined by the isomorphism class of a discrete uniform subgroup $\Gamma$. Among other things, we show that if $G$ has only finitely many components and $K$ is a maximal compact subgroup then $\Gamma$ determines the dimension of the space $G / K$. We then specialize our results to the case where $G / K$ is a riemannian symmetric space.
\end{abstract}

THEOREM. Let $G_{1}$ and $G_{2}$ be locally compact groups each with only finitely many components. Let $K_{i}$ be a maximal compact subgroup in $G_{i}$, let $X_{i}=G_{i} / K_{i}$ coset space, and let $\Gamma_{i}$ be a discrete subgroup of $G_{i}$.

(1) $X_{i}$ has a unique $G_{i}$-invariant structure of finite dimensional manifold.

(2) If $\Gamma_{i}$ is uniform in $G_{i}(i=1,2)$, and if $\Gamma_{1}$ and $\Gamma_{2}$ have subgroups of finite index that are isomorphic, then $\operatorname{dim} X_{1}=\operatorname{dim} X_{2}$.

(3) If $\operatorname{dim} X_{1}=\operatorname{dim} X_{2}, \Gamma_{1}$ is uniform in $G_{1}$, and $\Gamma_{1}$ and $\Gamma_{2}$ have subgroups of finite index that are isomorphic, then $\Gamma_{2}$ is uniform in $G_{2}$.

Proof. Let $U_{i}$ be an open neighborhood of 1 in $G_{i}$ and $L_{i}$ maximal among the compact normal subgroups of $G_{i}$ contained in $U_{i}$. Then $G_{i} / L_{i}$ has no small subgroups, hence is a Lie group, and $X_{i}$ $=\left(G_{i} / L_{i}\right) /\left(K_{i} / L_{i}\right)$. Statement (1) follows.

$U_{i}$ may be chosen so that $U_{i} \cap \Gamma_{i}=\{1\}$. Then $\Gamma_{i}$ projects isomorphically onto a discrete subgroup of the Lie group $G_{i} / L_{i}$. In proving (2) and (3) now we may assume that the $G_{i}$ are Lie groups. We may also replace $G_{i}$ by its identity component $G_{i}^{0}$ and $\Gamma_{i}$ by $\Gamma_{i} \cap G_{i}^{0}$. We may further cut the $\Gamma_{i}$ down to their subgroups of finite index that are isomorphic.

Now $G_{i}$ is a connected Lie group, $\Gamma_{i}$ is a discrete subgroup, and $\Gamma_{1} \cong \Gamma_{2}$.

Assume $\Gamma_{1}$ uniform in $G_{1}$. Then $\Gamma_{1}$, hence also $\Gamma_{2}$, is finitely generated. Let $\pi_{i}$ denote the adjoint representation of $G_{i}$, so $\operatorname{Ker}\left(\pi_{i}\right)$ is the center of $G_{i}$ and $A_{i}=\Gamma_{i} \cap \operatorname{Ker}\left(\pi_{i}\right)$ is a discrete central subgroup of $G_{i}$.

Received by the editors October 13, 1970.

AMS 1970 subject classifications. Primary 22E40.

Key words and phrases. Discrete subgroup, locally compact group, Lie group, symmetric space.

${ }^{1}$ Research partially supported by NSF Grant GP-8007.

Copyright @ 1971, American Mathematical Society 
Now $A_{i}$ is a finitely generated abelian subgroup of $\Gamma_{i}$ that is central in $G_{i} . \Gamma_{i} / A_{i}=\pi_{i}\left(\Gamma_{i}\right)$ is a finitely generated real linear group; so $[2$, Lemma 8$]$ it has a torsion free subgroup of finite index. Cut the $\Gamma_{i}$ down to isomorphic subgroups of finite index so that the $\Gamma_{i} / A_{i}$ are torsion free. Now $A_{i}$ is a finitely generated central subgroup of $\Gamma_{i}$ that contains every torsion element. Thus the torsion elements of $\Gamma_{i}$ form a finite abelian group $\Sigma_{i}$ that is central in $G_{i}$. Note $\Sigma_{1} \cong \Sigma_{2}$ and $\Gamma_{1} / \Sigma_{1} \cong \Gamma_{2} / \Sigma_{2}$. Now replace $G_{i}$ by $G_{i} / \Sigma_{i}, K_{i}$ by $K_{i} / \Sigma_{i}$ and $\Gamma_{i}$ by $\Gamma_{i} / \Sigma_{i}$. In summary, we may assume the $\Gamma_{i}$ torsion free.

$\Gamma_{i}$ is discrete in $G_{i}$ and torsion free, and $K_{i}$ is compact; so $\Gamma_{i}$ acts freely and properly discontinuously on $X_{i}=G_{i} / K_{i}$. Thus we have covering spaces $X_{i} \rightarrow Q_{i}=\Gamma_{i} \backslash X_{i} . X_{i}$ is acyclic so $X_{i} \rightarrow Q_{i}$ is a universal $\Gamma_{i}$-bundle. As $\Gamma_{1} \cong \Gamma_{2}$ now $Q_{1}$ and $Q_{2}$ are homotopy equivalent. Also, the $Q_{i}$ are manifolds.

Let $n_{i}$ be the smallest integer such that $H^{q}\left(Q_{i} ; Z_{2}\right)=0$ for $q>n_{i}$. Then $n_{1}=n_{2}$ because the $Q_{i}$ are homotopy equivalent. $Q_{1}$ is compact because $\Gamma_{1}$ is uniform in $G_{1}$; thus also $n_{1}=\operatorname{dim} Q_{1}$.

If $\Gamma_{2}$ is uniform in $G_{2}$ then $Q_{2}$ is compact and $n_{2}=\operatorname{dim} Q_{2}$. In that case $\operatorname{dim} X_{1}=\operatorname{dim} Q_{1}=n_{1}=n_{2}=\operatorname{dim} Q_{2}=\operatorname{dim} X_{2}$. Statement (2) is proved.

If $\operatorname{dim} X_{1}=\operatorname{dim} X_{2}$ then $\operatorname{dim} Q_{2}=\operatorname{dim} X_{2}=\operatorname{dim} X_{1}=\operatorname{dim} Q_{1}=n_{1}=n_{2}$. Whenever $Q$ is a noncompact $n$-manifold we know (put $q=0, A=Q$, $B=\varnothing$ and $G=Z_{2}$ in $\left[3\right.$, Theorem 6.4]) that $H^{n}\left(Q ; Z_{2}\right)=0$. Thus $Q_{2}$ is compact; so $\Gamma_{2}$ is uniform in $G_{2}$. Statement (3) is proved.

REMARK. The following fact was seen in the proof. Let $G$ be a locally compact group with only finitely many components and $\Gamma$ a finitely generated discrete subgroup. Then $\Gamma$ has a subgroup $\Delta$ of finite index, and $\Delta$ has a finite central subgroup $\Sigma$, such that $\Delta / \Sigma$ is torsion free.

We specialize to the semisimple case. The dimension statement in the following corollary is a special case of a forthcoming result of G. D. Mostow [1]. It is useful by itself and we supply an independent short proof.

Corollary. Let $G_{i}(i=1,2)$ be connected semisimple Lie groups with finite center, $K_{i}$ a maximal compact subgroup of $G_{i}$, and $\Gamma_{i}$ a discrete uniform subgroup of $G_{i}$. Suppose that $\Gamma_{1}$ and $\Gamma_{2}$ have subgroups of finite index that are isomorphic. Then the symmetric spaces $X_{i}=G_{i} / K_{i}$ of noncompact type satisfy

$\operatorname{rank} X_{1}=\operatorname{rank} X_{2}$ and $\operatorname{dim} X_{1}=\operatorname{dim} X_{2}$. 
In other words, real ranks and dimensions satisfy

$\operatorname{rank}_{R} G_{1}=\operatorname{rank}_{R} G_{2}$ and $\operatorname{dim} G_{1}-\operatorname{dim} K_{1}=\operatorname{dim} G_{2}-\operatorname{dim} K_{2}$.

INDEPENDENT SHORT PROOF. Passing to subgroups of finite index we may assume $[2$, Lemma 8$]$ that the $\Gamma_{i}$ are torsion free and isomorphic. As the $X_{i}$ are acyclic now the $p_{i}: X_{i} \rightarrow Q_{i}=\Gamma_{i} \backslash X_{i}$ are universal $\Gamma_{i}$-bundles, and $\Gamma_{1} \cong \Gamma_{2}$ shows the $Q_{i}$ homotopy equivalent. But the $Q_{i}$ are compact, so $\operatorname{dim} X_{i}=\operatorname{dim} Q_{i}$ is the smallest integer $n_{i}$ such that $H^{q}\left(Q_{i} ; Z_{2}\right)=0$ for $q>n_{i}$. Thus $\operatorname{dim} X_{1}=\operatorname{dim} X_{2}$.

We know [4, Theorem 4.2] that $r_{i}=\operatorname{rank}_{R} G_{i}$ is the maximum of the ranks of the free abelian subgroups of $\Gamma_{i}$, so $\operatorname{rank}_{R} G_{1}=\operatorname{rank}_{R} G_{2}$. However rank $X_{i}=\operatorname{rank}_{R} G_{i}$, so rank $X_{1}=\operatorname{rank} X_{2}$.

\section{REFERENCES}

1. G. D. Mostow, Results announced at the International Congress of Mathematicians, Nice, 1970 (to appear).

2. A. Selberg, On discontinuous groups in higher-dimensional symmetric spaces, Internat. Colloq. Function Theory, Contributions to Function Theory (Bombay, 1960), Tata Inst. of Fundamental Research, Bombay, 1960, pp. 147-164. MR 24 \#A188.

3. E. Spanier, Duality in topological manifolds, Colloque de Topologie (Brussels, 1964), Librairie Universitaire, Louvain, 1966, pp. 91-111. MR 36 \#3363.

4. J. A. Wolf, Discrete groups, symmetric spaces, and global holonomy, Amer. J. Math. 84 (1962), 527-542. MR 26 \#5523.

University of California, Berkeley, California 94720 